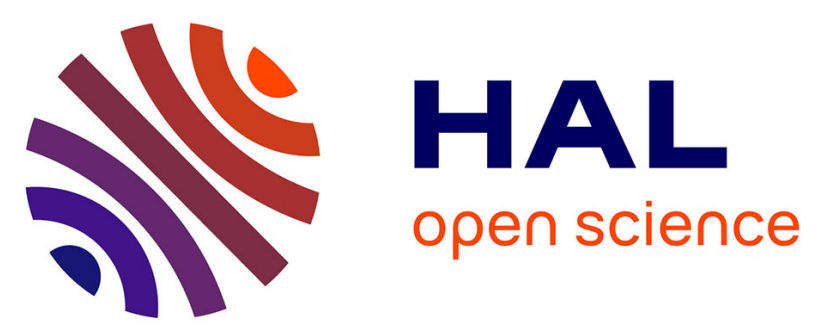

\title{
Choice between semi-parametric estimators of Markov and non-Markov multi-state models from coarsened observations
}

\author{
Daniel Commenges, Pierre Joly, Anne Gégout-Petit, Benoit Liquet
}

\section{- To cite this version:}

Daniel Commenges, Pierre Joly, Anne Gégout-Petit, Benoit Liquet. Choice between semi-parametric estimators of Markov and non-Markov multi-state models from coarsened observations: Choice between semi-parametric estimators of Markov and non-Markov multi-state models. Scandinavian Journal of Statistics, 2007, 34, pp.33-52. 10.1111/j.1467-9469.2006.00536.x inserm-00133006

\section{HAL Id: inserm-00133006 https://www.hal.inserm.fr/inserm-00133006}

Submitted on 26 Feb 2007

HAL is a multi-disciplinary open access archive for the deposit and dissemination of scientific research documents, whether they are published or not. The documents may come from teaching and research institutions in France or abroad, or from public or private research centers.
L'archive ouverte pluridisciplinaire $\mathbf{H A L}$, est destinée au dépôt et à la diffusion de documents scientifiques de niveau recherche, publiés ou non, émanant des établissements d'enseignement et de recherche français ou étrangers, des laboratoires publics ou privés. 


\section{Choice between semi-parametric estimators of Markov and non-Markov multi-state models from coarsened observations}

Daniel Commenges ${ }^{1,2}$, Pierre Joly ${ }^{1,2}$, Anne Gégout-Petit ${ }^{2}$, Benoit Liquet ${ }^{3}$

1 INSERM, U 875, Bordeaux, F33076, France

2 Université Victor Segalen Bordeaux 2, Bordeaux, F33076, France

3 Université de Grenoble, F33000, Grenoble

Running title: Choice between semi-parametric estimators

ABSTRACT. We consider models based on multivariate counting processes, including multi-state models. These models are specified semi-parametrically by a set of functions and real parameters. We consider inference for these models based on coarsened observations, focusing on families of smooth estimators such as produced by penalized likelihood. An important issue is the choice of model structure, for instance the choice between a Markov and some non-Markov models. We define in a general context the expected Kullback-Leibler criterion and we show that the likelihood based cross-validation $(L C V)$ is a nearly unbiased estimator of it. We give a general form of an approximate of the leave-one-out $L C V$. The approach is studied in simulation and illustrated by estimating Markov and two semi-Markov illness-death models with application on dementia using data of a large cohort study.

Key Words: counting processes, cross-validation, dementia, interval-censoring, Kullback-Leibler loss, Markov models, multi-state models, penalized likelihood, semi-Markov models. 


\section{Introduction}

Multi-state models, and more generally models based on multivariate counting processes, are well adapted for modeling complex event histories (Andersen et al., 1993; Hougaard, 2000). Assumptions have to be made about the law of the processes involved. In particular the Markov assumption has been made in many applications (Aalen \& Johansen, 1978; Joly et al., 2002) while semi-Markov models have been considered in other applications (Joly \& Commenges, 1999). Subject-matter knowledge can be a guide for making these assumptions (for instance risk of AIDS essentially depends on time since infection, leading Joly \& Commenges (1999) to choose a semi-Markov model); however in many cases the choice is not obvious. Other assumptions have to be made relative to the influence of explanatory variables: multiplicative or additive structures for instance may be considered. The problem is generally not to assert whether the "true" model is Markov or has a multiplicative structure but to choose the best model relative to the data at hand.

The semi-parametric approaches offer the greatest flexibility. Aalen (1978) has studied non-parametric inference for counting processes. If we wish to estimate smooth intensities we have to consider families of estimators such as kernel estimators (Ramlau-Hansen, 1983), sieve-estimators (Kooperberg \& Clarkson, 1997) or penalized likelihood estimators (Good \& Gaskin, 1971; O'Sullivan, 1988; Joly et al., 2002). These families are indexed by a parameter that we may call "smoothing coefficient". A practical way for choosing the smoothing coefficient is by optimizing a cross-validation criterion. In particular likelihood cross-validation $(L C V)$ has been shown to have good properties in simulation (Liquet, Sakarovitch \& Commenges, 2003; Liquet \& Commenges, 2004) while it has been shown that in some cases it could be considered as a proxy for the expected Kullback-Leibler loss and had the optimal property of being asymptotically as efficient as this theoretical criterion (Hall, 1987; van der Laan, Dudoit \& Keles, 2004). Liquet, Saracco \& Commenges (2006) argued that $L C V$ could be used not only for choosing the smoothing coefficient but also for choosing between different semi-parametric models, such as stratified and non-stratified proportional hazard survival model.

Additional complexity comes from the fact that the model must be estimated from incomplete data. Coarsening mechanisms have been studied in a general context by Gill, van der Laan \& Robins (1997). Commenges \& Gégout-Petit (2005) have studied a general time-coarsening model for pro- 
cesses which they called GCMP; we will use this coarsening process under the name TCMP for "time-coarsening model for processes"; in effect it is not completely general because it assumes that there are times where the process is exactly observed; this is most often the case for counting processes but not for more general processes. Even for counting processes the TCMP does not include the filtering process of Andersen et al. (1993) in a natural way. Writing the likelihood for observations of multi-state models through the TCMP has been done by Commenges \& Gégout-Petit (2006).

The aim of this paper is to advocate the use of the expected KullbackLeibler risk, $E K L$, based on the observation, for the choice between semiparametric estimators for coarsened observations. We also advocate the use of $L C V$ as an estimator of $E K L$. Thus $L C V$ can be used in particular for choosing between estimators of Markov and non-Markov multi-state models or between multiplicative and additive models in the presence of generally coarsened observations. It is worth noting that the $L C V$ choice fits well with using families of smooth estimators, such as produced by penalized likelihood, because non smooth estimators are strongly rejected by this criterion.

In section 2 we recall the description of multi-state models as multivariate counting processes and suggest possible Markov and non-Markov structures for the illness-death model. In section 3 we recall the construction of the likelihood ratio for counting processes and its extension to penalized likelihood and we unify the problem of choice of smoothing coefficients and model structure. In section 4 we tackle the problem of likelihood ratio and penalized likelihood in the TCMP framework. In section 5 we define the expected Kullback-Leibler loss as a general criterion for choosing an estimator in a family of estimators based on generally coarsened observations; we also study the case where there are observed explanatory variables. In section 6 it is proposed to use $L C V$ as a proxy for this theoretical criterion and we give a general approximation of the Leave-one-out $L C V$. In section 7 we present a simulation study in which we study in particular the variability of $L C V$ and we give insight about the interpretation of a difference of $L C V$. This approach is then applied in section 8 for choosing and estimating an illness-death model for dementia, based on the data of a large cohort study; section 9 concludes. 


\section{Multi-state and counting processes models; illness-death model}

\subsection{Multi-state and counting processes models}

A multi-state process $X=\left(X_{t}\right)$ is a right-continuous process which can take a finite number of values $\{0,1, \ldots, K\}$. If the model is Markov it can be specified by the transition intensities $\alpha_{h j}(), h,. j=0, \ldots, K$. The correspondence between multi-state processes and multivariate counting processes was studied in Commenges \& Gégout-Petit (2006) where the advantage of representing multi-state processes as multivariate "basic" counting processes is highlighted. Multi-state models are often generated by $p$ types of events, each type occurring just once. For instance the three-state illness-death model (see Figure 1) is generated by considering the events "illness" and "death" ; the five-state model considered by Commenges \& Joly (2004) is generated by "dementia", "institutionalization" and "death". So these multi-state models can be represented by a $p$-variate counting process $N$, each $N_{j}$ making at most one jump, and we will denote $T_{j}$ the jump time of $N_{j}$.

\subsection{Possible semi-parametric Models}

A model for a multivariate counting process $N=\left(N_{j}, j=1, \ldots, p\right)$ is specified for a given filtration $\left\{\mathcal{F}_{t}\right\}$ by its intensity $\lambda^{\theta}(t)=\left(\lambda_{j}^{\theta}(t), j=1 \ldots, p\right)$ under $P_{\theta}$.

For efficient inference one has to make assumptions: often the Markov assumption is made: the process is Markov if and only if $\lambda^{\theta}(t)$ is a function of only time $t$ and the indicator functions $1_{\left\{T_{j}<t\right\}}, j=1, \ldots, p$. An interesting non-Markov model occurs if $\lambda^{\theta}(t)$ depends on the time elapsed since the last jump of one of the components of $N$. If the intensities do not depend on the time $t$ itself this defines a particular semi-Markov model (used for instance by Lagakos, Sommer \& Zelen, 1978) that we will call "current-state" model because the transition intensities depend only on the time spent in the current state.

Completely parametric models are often too rigid but parametric assumptions may be made for some parts of the model: thus a semi-parametric approach, in which a great flexibility is preserved on some part of the model while some parametric assumptions are made for simplicity and easier interpretation, is often attractive. In such an approach, $\lambda^{\theta}(t)$ will depend on a 
certain number of functions on which no parametric assumptions are made, some of them representing baseline transition intensity functions, and parameters which appear in modeling how these baseline intensities will be changed as a function of events that have happened.

Let us consider some possible three-state irreversible illness-death models; these models are Markov or semi-Markov. Any model of this type is described by a bivariate counting process, $N_{1}$ counting illness and $N_{2}$ counting death. The intensity of $N_{1}$ necessarily takes the form $\lambda_{1}^{\theta}(t)=1_{\left\{T_{1} \geq t\right\}} 1_{\left\{T_{2} \geq t\right\}} \alpha_{01}(t)$, where $\alpha_{01}(t)$ has the interpretation of the transition intensity toward illness. The intensity of $N_{2}$ can generally be written $\lambda_{2}^{\theta}(t)=1_{\left\{T_{2} \geq t\right\}}\left[1_{\left\{T_{1} \geq t\right\}} \alpha_{02}(t)+\right.$ $\left.1_{\left\{T_{1}<t\right\}} \alpha_{12}\left(t, t-T_{1}\right)\right]$. The function $\alpha_{02}(t)$ has the interpretation of the transition intensity from health toward death (the mortality rate of healthy subjects). To avoid having to estimate non-parametrically a bivariate function we may consider models in which $\alpha_{12}\left(t, t-T_{1}\right)$ depends on two univariate functions $h(t)$ and $g\left(t-T_{1}\right)$; for instance we may consider an additive model $\alpha_{12}\left(t, t-T_{1}\right)=h(t)+g\left(t-T_{1}\right)$ as in Scheike (2001).

Particular cases of this model are:

$\mathcal{M}_{1}: g=0$ : non-homogeneous Markov model; here $h(t)$ has the interpretation of $\alpha_{12}(t)$, the transition intensity from illness toward death;

$\mathcal{M}_{2}: h(t)=0$ : current state model; here $g($.$) has the interpretation of a$ random transition intensity from illness toward death;

$\mathcal{M}_{3}: h(t)=\alpha_{02}(t)$ : excess mortality model; here $g\left(t-T_{1}\right)$ has the interpretation of an excess mortality due to illness as a function of time passed in the illness state.

If explanatory variables $Z_{i}(t)$ for subject $i$ are available we may consider different models for the dependence of the intensities on the $Z_{i}($.$) (the vari-$ ables are either external or internal and in the latter case the filtration must be rich enough so that the processes $\left(Z_{i}(t)\right)$ be adapted); in particular a multiplicative structure (in the spirit of the proportional hazard model) or an additive structure (in the spirit of the Aalen additive model: Aalen, Borgan \& Fekjær, 2001) could be considered. For instance a multiplicative structure for the explanatory variables could be:

$$
\begin{aligned}
\alpha_{01}^{i}(t) & =\alpha_{01}^{0}(t) \exp \left(\beta_{01} Z_{i}(t)\right), \\
\alpha_{02}^{i}(t) & =\alpha_{02}^{0}(t) \exp \left(\beta_{02} Z_{i}(t)\right), \\
\alpha_{12}^{i}\left(t, t-T_{1}\right) & =\alpha_{12}^{0}\left(t, t-T_{1}\right) \exp \left(\beta_{12} Z_{i}(t)\right),
\end{aligned}
$$


where $\alpha_{01}^{0}(t), \alpha_{02}^{0}(t)$ and $\alpha_{12}^{0}\left(t, t-T_{1}\right)$ are baseline transition intensities (the last one being generally random and in that case defined only on $\left\{t>T_{1}\right\}$ ).

\section{Likelihood and penalized likelihood for count- ing processes}

\subsection{Likelihood ratios}

The model specifies a family of probability measures $\left\{P_{\theta}\right\}_{\theta \in \Theta}$; consider a reference probability measure $P_{0}$ such that each $P_{\theta}$ is absolutely continuous relatively to $P_{0}\left(P_{0}\right.$ may or may not belong to $\left.\left\{P_{\theta}\right\}_{\theta \in \Theta}\right)$. The likelihood ratio on a $\sigma$-field $\mathcal{X}$ is defined by:

$$
\mathcal{L}_{\mathcal{X}}^{P_{\theta} / P_{0}}=\frac{d P_{\theta}}{d P_{0} \mid \mathcal{X}} \quad \text { a.s. }
$$

where $\frac{d P_{\theta}}{d P_{0} \mid \mathcal{X}}$ is the Radon-Nikodym derivative of $P_{\theta}$ relatively to $P_{0}$ on $\mathcal{X}$.

Remark 1. All equalities involving likelihood ratios or conditional expectations are a.s. equalities; this may not be recalled every time.

Remark 2. Often likelihoods are computed using a reference measure that is not a probability measure and is not even specified. Here we will make it explicit and take a probability measure, in which case the term "likelihood ratio" is warranted. If the reference probability $P_{0}$ belongs to $\left(P_{\theta}\right)_{\theta \in \Theta}$ then there exist $\theta_{0}$ such that $P_{0}=P_{\theta_{0}}$ and we may write $\mathcal{L}_{\mathcal{X}}^{\theta / \theta_{0}}=\mathcal{L}_{\mathcal{X}}^{P_{\theta} / P_{0}}$.

One of the advantages of representing multi-state models in the framework of counting processes (such in as section 2.1) is the availability of Jacod's formula for the likelihood ratio based on observation on $[0, C]$ in the filtration $\left\{\mathcal{G}_{t}\right\}$ where $\mathcal{G}_{t}=\mathcal{G}_{0} \vee \mathcal{N}_{t}$, where $\mathcal{N}_{t}=\sigma\left(N_{j u}, 0 \leq u \leq t\right)$. The model is specified by the intensities $\lambda_{j}^{\theta}(t)$ of the $N_{j}$ 's under $P_{\theta}$. It is advantageous to take as reference probability, a probability $P_{0}$ under which the $N_{j}$ 's are independent with intensities $\lambda_{j}^{0}(t)=1_{\left\{N_{j t-}=0\right\}}$; equivalently the $T_{j}$ 's are independent with exponential distributions with unit parameter. Using Jacod's formula (Jacod, 1975) the likelihood ratio for this reference probability is:

$$
\mathcal{L}_{\mathcal{G}_{C}}^{P_{\theta} / P_{0}}=\mathcal{L}_{\mathcal{G}_{0}}^{P_{\theta} / P_{0}} \prod_{r=1}^{N_{. C}} \lambda_{J_{r}}^{\theta}\left(T_{(r)}\right) \exp \left(-\Lambda_{.}^{\theta}(C)\right) \prod_{j=1}^{p} e^{T_{j} \wedge C},
$$


where for each $r \in\left\{1, \ldots, N_{. C}\right\}, J_{r}$ is the unique $j$ such that $\Delta N_{j T_{(r)}}=1$; $N_{. t}=\sum_{j=1}^{p} N_{j t}, \Lambda_{.}^{\theta}(t)=\sum_{j=1}^{p} \Lambda_{j}^{\theta}(t), \Lambda_{j}^{\theta}(t)=\int_{0}^{t} \lambda_{j}^{\theta}(u) d u$. This formula allows us to compute the likelihood for any multi-state model once we have written it as a multivariate counting process.

\subsection{Families of penalized likelihood estimators}

Consider models specified by a set of parameters $\theta=(g, \beta)$ where $g()=$. $\left(g_{j}(),. j=1, \ldots, K\right)$ is a vector of functions from $\Re$ to $\Re$ and $\beta$ a vector of real parameters. For instance for the Markov illness-death model $\theta=\left(\alpha_{01}(),. \alpha_{02}(),. \alpha_{12}(),. \beta\right)$ where the $\alpha_{h j}$ are transition intensities and $\beta$ is a vector of regression coefficients. If no parametric assumptions are made about the functions to be estimated and if smooth estimators are favored, the two main approaches are sieve estimators, extending the so-called hazard regression of Kooperberg \& Clarkson (1997) or using orthogonal expansions such as in Müller \& Stadtmüller (2005), and penalized likelihood (Gu, 1996; Joly et al., 2002).

Suppose that the sample consists of $n$ independent observations of multivariate counting processes $N^{i}=\left(N_{j}^{i}, j=1, \ldots, p\right), i=1, \ldots, n$ represented by $\mathcal{G}_{i C_{i}}$; the likelihood ratio $\mathcal{L}_{\overline{\mathcal{O}}_{n}}^{P_{\theta} / P_{0}}$, where $\overline{\mathcal{O}}_{n}=\vee \mathcal{G}_{i C_{i}}$, is the product of contributions computed with formula (1). A penalized log-likelihood is defined as:

$$
p l_{\overline{\mathcal{O}}_{n}}^{\kappa}(\theta)=\log \mathcal{L}_{\overline{\mathcal{O}}_{n}}^{P_{\theta} / P_{0}}-J(\theta, \kappa),
$$

where $\kappa=\left(\kappa_{j}, j=1, \ldots, K\right)$ is a set of smoothing coefficients. It is common to use a penalty based on the $L_{2}$-norms of the second derivatives of the unknown functions:

$$
J(g(.), \kappa)=\sum_{j=1}^{K} \kappa_{j} \int\left(g_{j}^{\prime \prime}\right)^{2}(u) d u
$$

The penalized likelihood defines a family of estimators of $\theta,\left(\hat{\theta}_{\kappa}\right)_{\kappa \in \Re{ }^{K}+}$, and thus a family of estimators of the probability $P_{\theta},\left(P_{\hat{\theta}_{\kappa}}\right)_{\kappa \in \Re^{K}+}$. Asymptotic results have been given for particular cases (Cox \& O'Sullivan, 1990; Gu, 1996; Eggermont \& La Ricia, 1999, 2001).

Consider now the situation where we can choose between different basic assumptions for our model (such as Markov or semi-Markov assumptions), indexed by $\eta=1, \ldots, m$. Formally we could include $\eta$ in $\theta$. However we prefer 
to formalize the problem in a way that is closer to intuition and practice: for each value of $\eta$ and each $\kappa$ we have a maximum penalized likelihood estimator $\hat{\theta}_{\kappa}^{\eta}$; thus we have a family of estimators of the probability specified by $\left(P_{\hat{\theta}_{\kappa}^{\eta}}\right)_{\eta=1, \ldots, m ; \kappa \in \Re K_{+}}$. The problem is to choose one estimator in this family. In the following we will include $\eta$ in $\kappa$ considering that $\kappa$ indexes a family of estimators, thus unifying the problem of smoothing coefficient and model structure.

\section{Penalized likelihood for coarsened at ran- dom counting processes}

\subsection{Coarsening at random in the TCMP}

Here we consider a general case of incomplete data: we recall the TCMP model proposed in Commenges \& Gégout-Petit (2005) and we give a version of the coarsening at random condition CAR(TCMP) and the factorization theorem it implies, adapted to the case where the reference probability is outside of the model; also we exhibit a "reduced" model that we will use in the sequel. The TCMP can be considered for any stochastic process $X$; when $X$ is a counting process the TCMP includes in particular extensions of the concepts of right-, left- and interval-censoring that have been defined for survival data, as well as a combination of these different types of censoring.

Definition 1 (The time coarsening model for processes (TCMP)) $A$ TCMP is a scheme of observation for a multivariate process $X=\left(X_{1}, \ldots, X_{p}\right)$ specified by a multivariate response process $R=\left(R_{1}, \ldots, R_{p}\right)$, where the $R_{j t}$ 's take values 0 or 1 for all $j$ and $t$, such that $X_{j t}$ is observed at time $t$ if and only if $R_{j t}=1$, for $j=1, \ldots, p$; that is, the observed $\sigma$-field is $\mathcal{O}=\sigma\left(R_{t}, R . X_{t}, t \geq 0\right)$.

In the definition we denote $R \cdot X_{t}=\left(R_{1 t} X_{1 t}, \ldots, R_{p t} X_{p t}\right)$. A model for $(X, R)$ is a family of measures $\left\{P_{\theta \psi}\right\}_{(\theta, \psi) \in \Theta \times \Psi}$ on a measurable space $(\Omega, \mathcal{F}) . X$ (resp. $R$ ) takes values in a measurable space $(\Xi, \xi)$ (resp. $(\Gamma, \rho))$. For us $X$ and $R$ will be p-dimensional càdlàg stochastic processes, so $(\Xi, \xi)$ and $(\Gamma, \rho)$ are Skorohod spaces endowed with their Borel $\sigma$-fields. The parameter spaces $\Theta$ and $\Psi$ need not be finite dimensional. We will assume that the measures in the family are equivalent. The processes $X$ and $R$ generate $\sigma$-fields $\mathcal{X}$ and $\mathcal{R}$, and we shall take $\mathcal{F}=\mathcal{X} \vee \mathcal{R} . P_{\theta \mathcal{X}}$ is the restriction of $P_{\theta \psi}$ to $\mathcal{X}$ : 
that is, the marginal probability of $X$ does not depend on $\psi$. The additional parameter $\psi$ will be considered as a nuisance parameter. We assume a "Non-Informativeness" assumption in the coarsening mechanism, which, writing $P_{\theta \psi}^{\mathcal{X}}=P_{\theta \psi}(. \mid \mathcal{X})$ (a conventional notation for conditional probabilities: Kallenberg, 2001), is :

$$
P_{\theta_{1} \psi}^{\mathcal{X}}=P_{\theta_{2} \psi}^{\mathcal{X}}, \text { a.s., for all } \theta_{1}, \theta_{2}, \psi
$$

This is a conventional assumption (although it has not been expressed in this form) and means that the coarsening mechanism (conditionally on $X$ ) depends on a distinct (from the parameter of interest $\theta$ ), "variation independent" parameter $\psi$. Now we consider a family of equivalent probabilities $\mathcal{Q}$ including in addition to $\left\{P_{\theta \psi}\right\}_{(\theta, \psi) \in \Theta \times \Psi}$ a family of possible reference probabilities. Let $P_{0}$ be one such probability; we denote by $P_{0 \mathcal{X}}$ its restriction to $\mathcal{X}$ and by $P_{0}^{\mathcal{X}}$ the associated conditional probability given $\mathcal{X}$. The likelihood ratio is then $\mathcal{L}_{\mathcal{F}}^{P_{\theta \psi} / P_{0}}=\mathcal{L}_{\mathcal{R} \mid \mathcal{X}}^{P_{\theta \psi} / P_{0}} \mathcal{L}_{\mathcal{X}}^{P_{\theta \mathcal{X}} / P_{0 \mathcal{X}}}$, where $\mathcal{L}_{\mathcal{R} \mid \mathcal{X}}^{P_{\theta \psi} / P_{0}}$ is the conditional likelihood of $\mathcal{R}$ given $\mathcal{X}$. Note that with the non-informativeness assumption $\mathcal{L}_{\mathcal{R} \mid \mathcal{X}}^{P_{\theta \psi} / P_{0}}$ does not depend on $\theta$; we will note it $\mathcal{L}_{\mathcal{R} \mid \mathcal{X}}^{P_{. \psi} / P_{0}}$ to emphasize this fact. $\mathcal{L}_{\mathcal{F}}^{P_{\theta \psi} / P_{0}}$ is the full likelihood and $\mathcal{L}_{\mathcal{O}}^{P_{\theta \psi} / P_{0}}$ the observed likelihood.

Definition 2 (CAR(TCMP)) We will say that CAR(TCMP) holds for the couple $(X, R)$ in $\mathcal{Q}$ if $\mathcal{L}_{\mathcal{R} \mid \mathcal{X}}^{P_{1} / P_{0}}$ is $\mathcal{O}$-measurable for all $P_{1}, P_{0} \in \mathcal{Q}$.

We will use the following result (which is an adaptation of Theorem 2 in Commenges \& Gégout-Petit, 2005):

Theorem 1 (Factorization) If the couple $(R, X)$ satisfies $C A R(T C M P)$ then we have $\mathcal{L}_{\mathcal{O}}^{P_{\theta \psi} / P_{0}}=\mathcal{L}_{\mathcal{R} \mid \mathcal{X}}^{P_{. \psi} / P_{0}} \mathrm{E}_{P_{0}}\left[\mathcal{L}_{\mathcal{X}}^{P_{\theta \mathcal{X}} / P_{0 \mathcal{X}}} \mid \mathcal{O}\right]$ and $\mathrm{E}_{P_{0}}\left[\mathcal{L}_{\mathcal{X}}^{P_{\theta \mathcal{X}} / P_{0 \mathcal{X}}} \mid \mathcal{O}\right]$ does not depend on $P_{0}^{\mathcal{X}}$.

This factorization is a first step toward ignorability because for instance it is the same value of $\theta$ which maximizes $\mathrm{E}_{P_{0}}\left[\mathcal{L}_{\mathcal{X}}^{P_{\theta \mathcal{X}} / P_{0 \mathcal{X}}} \mid \mathcal{O}\right]$ and which maximizes $\mathcal{L}_{\mathcal{O}}^{P_{\theta \psi} / P_{0}}$; a slightly stronger condition is necessary for ignorability (see Commenges \& Gégout-Petit, 2005).

We can achieve a nicer result which will help simplifying notations in section 5. If we knew the true conditional probability given $\mathcal{X}, P_{*}^{\mathcal{X}}$, we would use the following "reduced" model: $\left\{P_{\theta}\right\}_{\theta \in \Theta}$ such that $P_{\theta}^{\mathcal{X}}=P_{*}^{\mathcal{X}}$. 
Definition 3 (Reduced model) Given a model $\left\{P_{\theta, \psi}\right\}_{(\theta, \psi) \in \Theta \times \Psi}$ such that the restriction of $P_{\theta, \psi}$ to $\mathcal{X}$ is $P_{\theta \mathcal{X}}$ for all $\theta$ and $\psi$, we call "reduced model " the model $\left\{P_{\theta}\right\}_{\theta \in \Theta}$ such as the restriction of $P_{\theta}$ to $\mathcal{X}$ is $P_{\theta \mathcal{X}}$ and $P_{\theta}^{\mathcal{X}}=P_{*}^{\mathcal{X}}$ for all $\theta$, where $P_{*}^{\mathcal{X}}$ is the true conditional probability given $\mathcal{X}$.

Note that this reduced model is "reduced" in the sense that it is a smaller family than the original one; however it is a submodel only if the original model was well-specified $\left(P_{*} \in\left\{P_{\theta, \psi}\right\}_{(\theta, \psi) \in \Theta \times \Psi}\right)$. Taking a reference probability $P_{0}$ such that $P_{0}^{\mathcal{X}}=P_{*}^{\mathcal{X}}$ we have that $\mathcal{L}_{\mathcal{R} \mid \mathcal{X}}^{P_{\theta} / P_{0}}=1$ a.s. Thus we have, without additional assumption, $\mathcal{L}_{\mathcal{O}}^{P_{\theta} / P_{0}}=\mathrm{E}_{P_{0}}\left[\mathcal{L}_{\mathcal{X}}^{P_{\mathcal{X}} / P_{0 \mathcal{X}}} \mid \mathcal{O}\right]$. If CAR(TCMP) holds $\mathrm{E}_{P_{0}}\left[\mathcal{L}_{\mathcal{X}}^{P_{\theta \mathcal{X}} / P_{0 \mathcal{X}}} \mid \mathcal{O}\right]$ does not depend on $P_{0}^{\mathcal{X}}=P_{*}^{\mathcal{X}}$. That is, we can compute the exact likelihood that we would like to compute if we knew $P_{*}^{\mathcal{X}}$, without in fact knowing it.

There remains in practice to compute $\mathrm{E}_{P_{0}}\left[\mathcal{L}_{\mathcal{X}}^{P_{\theta \mathcal{X}} / P_{0 \mathcal{X}}} \mid \mathcal{O}\right]$ for the observed value of $R$ and this is explained for the case of multistate processes in the next section.

\subsection{Likelihood and penalized likelihood for counting processes}

The likelihood for the deterministic TCMP (that is in which the $R$ 's are deterministic functions) and when $X$ is a multivariate counting process has been given in Commenges \& Gégout-Petit (2006). The observation in this scheme is denoted by the $\sigma$-field $\mathcal{O}$ and we have $\mathcal{O} \subset \mathcal{G}_{C}$, so that the observed likelihood can be expressed as: $\mathcal{L}_{\mathcal{O}}^{P_{\theta} / P_{0}}=\mathrm{E}_{P_{0}}\left[\mathcal{L}_{\mathcal{G}_{\mathcal{C}}}^{P_{\theta} / P_{0}} \mid \mathcal{O}\right]$. The formula for the likelihood follows from computation of this conditional expectation using the disintegration theorem (Kallenberg, 2001).

In the case of the stochastic TCMP when CAR(TCMP) holds we use the reduced model $\left\{P_{\theta}\right\}_{\theta \in \Theta}$ so that the likelihood ratio is $\mathcal{L}_{\mathcal{O}}^{P_{\theta} / P_{0}}=\mathrm{E}_{P_{0}}\left(\mathcal{L}_{\mathcal{G}_{\mathcal{C}}}^{P_{\theta} / P_{0}} \mid \mathcal{O}\right)$ and $\mathrm{E}_{P_{0}}\left(\mathcal{L}_{\mathcal{G}_{\mathcal{C}}}^{P_{\theta} / P_{0}} \mid \mathcal{O}\right)$ does not depend on $P_{0}^{\mathcal{X}}$; with a slightly stronger condition it can be computed in practice as if the TCMP was deterministic, using the formulae given in Commenges \& Gégout-Petit (2006), with values of the responses functions $r$ equal to what has been observed.

As for the penalized likelihood it can also be extended to the case where the observations are CAR(TCMP): the formula is the same as (2). 


\section{Choice between semi-parametric models: ex- pected Kullback-Leibler loss}

\subsection{General theory}

The problem of choice of an estimator among a family of estimators using expected Kullback-Leibler risk has been studied in particular by Hall (1987), van der Laan \& Dudoit (2003), van der Laan, Dudoit \& Keles (2004). Since our aim here is to choose an estimator of a probability measure among a family of estimators, this theoretical criterion is particularly relevant. We formalize this criterion in this general context and for incomplete observations. Although the focus of the paper is on counting processes, the formalism developed in this section applies to more general processes, so we will call the process of interest $X$ as in section 4.1.

Let us now model $n$ i.i.d. random elements $\left(X_{i}, R_{i}\right), i=1, \ldots, n$. We consider a measurable space $\left(\bar{\Omega}_{n}, \overline{\mathcal{X}}_{n}, \overline{\mathcal{R}}_{n}\right)$ where $\bar{\Omega}_{n}=\times \Omega_{i}, \overline{\mathcal{X}}_{n}=\otimes \mathcal{X}_{i}, \overline{\mathcal{R}}_{n}=$ $\otimes \mathcal{R}_{i}$ where the $\sigma$-fields for different $i$ are independent. The probability measures on this space are the product measures. Finally we define full and observed $\sigma$-fields $\mathcal{F}_{i}=\mathcal{R}_{i} \vee \mathcal{X}_{i}$ and $\mathcal{O}_{i}=\sigma\left(R_{i t}, R_{i t} . X_{i t}, t \geq 0\right)$ respectively.

The problem is to estimate $\theta$. When $\Theta$ is a functional space a conventional strategy is to define estimators (that is $\overline{\mathcal{O}}_{n}$-measurable functions) depending on a meta-parameter $\kappa: \hat{\theta}\left(\kappa, \overline{\mathcal{O}}_{n}\right)$. $\kappa$ may index nested models such as in sieve estimators or may be a smoothing parameter such as in penalized likelihood or kernel estimators. According to statistical decision theory (Le Cam \& Yang, 1990) we should choose an estimator which minimizes a risk function, the expectation of a loss function. In statistics we assume that there is a true probability $P_{*}$. We do not make the assumption that $P_{*}$ belongs to the model; this assumption would significantly reduce the scope of the theory. Making the assumption that $P_{*}$ is equivalent to the probabilities of the model, the most natural "all-purpose" loss function relatively to $P_{*}$ is $-\log \mathcal{L}_{\mathcal{X}_{n+1}}^{P_{\hat{\mathcal{X}}} / P_{* \mathcal{X}}}$ (we note $\hat{\theta}=\hat{\theta}\left(\kappa, \overline{\mathcal{O}}_{n}\right)$ ), where $P_{* \mathcal{X}}$ is the restriction of $P_{*}$ to $\mathcal{X}$. However the problem will be to "estimate" the risk, that is to find a statistic $\left(\overline{\mathcal{O}}_{n}\right.$-measurable) which takes values close to that risk (this is not exactly an estimation problem because the target moves with $n$ but we will use the word "estimate" for simplicity). It may be considered as intuitive that a risk based on $-\log \mathcal{L}_{\mathcal{X}_{n+1} P_{*} / P_{* \mathcal{X}}}^{P_{n}}$ will be very difficult to estimate; this is why Liquet and Commenges (2004) suggested using the expectation of the 
observed loglikelihood of the sample, a criterion they denoted ELL. The use of the stochastic TCMP and the CAR(TCMP) assumption allows us working in the more comfortable i.i.d. framework for incomplete data leading to more elegant and general results.

The straightforward loss function on $\mathcal{O}_{n+1}$ is $-\log \mathcal{L}_{\mathcal{O}_{n+1}, \hat{\psi}}^{P_{\hat{\theta}} / P_{*}}$. A difficulty arises in that this loss function requires an estimator of $\psi$. Hopefully the CAR(TCMP) assumption allows us to construct a reduced model as in section 4.1 in which we can construct a loss function not depending on the conditional probability given $\mathcal{X}, P_{*}^{\mathcal{X}}$. The first step is to construct the reduced model associated to the reference probability $P_{*}$. Then we have, by applying the result of section 4.1 to that case and to $\sigma$-fields $\mathcal{X}_{n+1}, \mathcal{R}_{n+1}, \mathcal{O}_{n+1}$ : $\mathcal{L}_{\mathcal{O}_{n+1}}^{P_{\theta} / P_{*}}=\mathrm{E}_{P_{*}}\left[\mathcal{L}_{\mathcal{X}_{n+1}}^{P_{\theta \mathcal{X}} / P_{* \mathcal{X}}} \mid \mathcal{O}_{n+1}\right]$ and this does not depend on $P_{*}^{\mathcal{X}}$. We can now

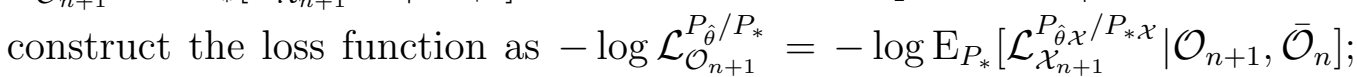
note that we must add the conditioning on $\overline{\mathcal{O}}_{n}$ because $\hat{\theta}$ is an $\overline{\mathcal{O}}_{n}$-measurable random variable.

The conditional expectation of this loss, or conditional risk, is $C K L_{n}=$ $\mathrm{E}_{P_{*}}\left[-\log \mathcal{L}_{\mathcal{O}_{n+1}}^{P_{\hat{\theta}} / P_{*}} \mid \overline{\mathcal{O}}_{n}\right]$ and can be interpreted as the Kullback-Leibler divergence between $P_{\hat{\theta}}$ and $P_{*}$, since the Kullback-Leibler divergence of a probability $P_{1}$ relatively to $P_{*}$ over the $\sigma$-field $\mathcal{O}_{n+1}$ is $K L\left(P_{1}, P_{*}\right)=\mathrm{E}_{P_{*}}\left[-\log \mathcal{L}_{\mathcal{O}_{n+1}}^{P_{1} / P_{*}}\right]$. Its expectation, or risk, $E K L_{n}=\mathrm{E}_{P_{*}}\left[-\log \mathcal{L}_{\mathcal{O}_{n+1}}^{P_{\hat{\theta}} / P_{*}}\right]$, can be interpreted as the expected Kullback-Leibler divergence over the $\sigma$-field $\mathcal{O}_{n+1}$ of interest.

\subsection{Case of observed explanatory variables}

Explanatory variables are considered as stochastic processes $Z=\left(Z_{t}\right)_{t \geq 0}$. We can then consider the process $W=(X, Z)$. In the TCMP framework we associate the response process $R=\left(R_{X}, R_{Z}\right)$. The observed $\sigma$-field is $\mathcal{O}=\sigma\left(R_{t}, R . W_{t}, t \geq 0\right)$. With such a formulation there is no need of a special theory for explanatory variables. However it is often the case that: i) the marginal law of $Z$ is not of interest, but only the conditional law of $X$ given $Z$ is of interest; ii) $Z$ is completely observed, that is, all the components of $R_{Z}$ are identically equal to one (we will refer to this by writing $R_{Z}=1$ ). Because of i) we consider the following parametrization of the model: the model is defined by the family of probability measures $\left(P_{\theta \gamma \psi}\right)_{\theta \in \Theta, \gamma \in \Gamma, \psi \in \Psi}$, where $P_{\theta \gamma \psi}$ is specified by $P_{\gamma \mathcal{Z}}, P_{\psi}^{\mathcal{X}, \mathcal{Z}}$ and $P_{\theta \mathcal{X}}^{\mathcal{Z}}$, that is $\gamma$ indexes the marginal probability of $Z, \psi$ the conditional probability given $\mathcal{X}$ and $\mathcal{Z}$, and $\theta$ the 
conditional probability of $X$ given $\mathcal{Z}$ on which the interest focuses. We take a reference probability $P_{0}$ and we assume that CAR(TCMP) holds for $(W, R)$, that is $\mathcal{L}_{\mathcal{R} \mid \mathcal{W}}^{P_{\theta \gamma \psi} / P_{0}}$ is $\mathcal{O}$-measurable; this is equivalent to $\mathcal{L}_{\mathcal{R}_{X} \mid \mathcal{W}}^{P_{\theta \gamma} / P_{0}} \mathcal{O}$-measurable (where $\mathcal{R}_{X}$ is the $\sigma$-field generated by $R_{X}$ ) because $R_{Z}=1$ (this can be seen for instance by applying property vi) of Commenges \& Gégout-Petit, 2005). In that case, if ii) holds, we can get rid of both nuisance parameters $\psi$ and $\gamma$ for the likelihood inference on $\theta$. This is a consequence of the double-factorization theorem.

Theorem 2 (Double-factorization) Consider the process $W=(X, Z)$, where $X$ is the process of interest, $Z$ is a process of explanatory variables; $R=$ $\left(R_{X}, R_{Z}\right)$ is the associated response process and we have $R_{Z}=1$. Consider the family of equivalent probability measures $\mathcal{Q}=\left\{\left\{P_{\theta \gamma \psi}\right\}_{\theta \in \Theta, \gamma \in \Gamma, \psi \in \Psi}, \mathcal{Q}_{0}\right\}$, where $P_{\theta \gamma \psi}$ is specified by $P_{\gamma \mathcal{Z}}, P_{\theta \mathcal{X}}^{\mathcal{Z}}$ and $P_{\psi}^{\mathcal{X}, \mathcal{Z}}$, and where $\mathcal{Q}_{0}$ is a family of possible reference probabilities; the restriction of $P_{\theta \gamma \psi}$ on $\mathcal{W}$ is denoted $P_{\theta \gamma \mathcal{W}}$. Consider a reference probability $P_{0} \in \mathcal{Q}_{0}$. If the couple $(W, X)$ satisfies $C A R(T C M P)$ in $\mathcal{Q}$, then we have:

$$
\mathcal{L}_{\mathcal{O}}^{P_{\theta \gamma \psi} / P_{0}}=\mathcal{L}_{\mathcal{R} \mid \mathcal{W}}^{P_{\theta \gamma \psi} / P_{0}} \mathcal{L}_{\mathcal{Z}}^{P_{\gamma \mathcal{Z}} / P_{0 \mathcal{Z}}} \mathrm{E}_{P_{0}}\left[\mathcal{L}_{\mathcal{X} \mid \mathcal{Z}}^{P_{\theta \gamma \mathcal{W}} / P_{0} \mathcal{W}} \mid \mathcal{O}\right]
$$

and

1. $\mathcal{L}_{\mathcal{R} \mid \mathcal{W}}^{P_{\theta \gamma \psi} / P_{0}}$ depends neither on $\theta$ nor on $\gamma$ and can be denoted $\mathcal{L}_{\mathcal{R} \mid \mathcal{W}}^{P^{.}{ }^{2} / P_{0}}$; $\mathcal{L}_{\mathcal{Z}}^{P_{\gamma \mathcal{Z}} / P_{0 \mathcal{Z}}}$ depends neither on $\theta$ nor on $\psi ; \mathcal{L}_{\mathcal{X} \mid \mathcal{Z}}^{P_{\theta \gamma \mathcal{W}} / P_{0 \mathcal{W}}}$ depends neither on $\psi$ nor on $\gamma$ and can be denoted $\mathcal{L}_{\mathcal{X} \mid \mathcal{Z}}^{P_{\theta . \mathcal{W}} / P_{0 \mathcal{W}}}$

2. $\mathrm{E}_{P_{0}}\left[\mathcal{L}_{\mathcal{X} \mid \mathcal{Z}}^{P_{\theta \cdot \mathcal{W}} / P_{0} \mathcal{W}} \mid \mathcal{O}\right]$ depends neither on $P_{0}^{\mathcal{X}, \mathcal{Z}}$ nor on $P_{0 \mathcal{Z}}$.

Proof. Since CAR(TCMP) holds for $(W, R)$ we can apply the (simple) factorization theorem which gives: $\mathcal{L}_{\mathcal{O}}^{P_{\theta \gamma \psi} / P_{0}}=\mathcal{L}_{\mathcal{R} \mid \mathcal{W}}^{P_{\theta \gamma \psi} / P_{0}} \mathrm{E}_{P_{0}}\left[\mathcal{L}_{\mathcal{W}}^{P_{\theta \gamma \mathcal{W}} / P_{0} \mathcal{W}} \mid \mathcal{O}\right]$. We next use the decomposition $\mathcal{L}_{\mathcal{W}}^{P_{\theta \gamma \mathcal{W}} / P_{0 \mathcal{W}}}=\mathcal{L}_{\mathcal{Z}}^{P_{\gamma \mathcal{Z}} / P_{0 \mathcal{Z}}} \mathcal{L}_{\mathcal{X} \mid \mathcal{Z}}^{P_{\theta \gamma \mathcal{W}} / P_{0 \mathcal{W}}}$ and since $\mathcal{L}_{\mathcal{Z}}^{P_{\gamma \mathcal{Z}} / P_{0 \mathcal{Z}}}$ is $\mathcal{O}$-measurable (because $\mathcal{L}_{\mathcal{Z}}^{P_{\gamma \mathcal{Z}} / P_{0 \mathcal{Z}}}$ is $\mathcal{Z}$-measurable and $\mathcal{Z} \subset \mathcal{O}$ ) we obtain (4). Point (1) is straightforward. Another way to express Point (2) is that if we consider two probabilities $P_{1}$ and $P_{0}$ such that $P_{0 \mathcal{X}}^{\mathcal{Z}}=P_{1 \mathcal{X}}^{\mathcal{Z}}$ we have $\mathrm{E}_{P_{0}}\left[\mathcal{L}_{\mathcal{X} \mid \mathcal{Z}}^{P_{\theta . \mathcal{W}} / P_{0} \mathcal{W}} \mid \mathcal{O}\right]=\mathrm{E}_{P_{1}}\left[\mathcal{L}_{\mathcal{X} \mid \mathcal{Z}}^{P_{\theta . \mathcal{W}} / P_{1 \mathcal{W}}} \mid \mathcal{O}\right]$. The proof is similar to that of Theorem 1 given in Commenges \& Gégout-Petit (2005).

We can now apply the trick of the reduced model of section 4.1 to this context. If we knew the true conditional probability given $(\mathcal{X}, \mathcal{Z}), P_{*}^{\mathcal{X}, \mathcal{Z}}$, and 
the true marginal probability $P_{* \mathcal{Z}}$, we would use the reduced model $\left\{P_{\theta}\right\}_{\theta \in \Theta}$ such that $P_{\theta}^{\mathcal{X}, \mathcal{Z}}=P_{*}^{\mathcal{X}, \mathcal{Z}}$ and $P_{\theta \mathcal{Z}}=P_{* \mathcal{Z}}$. Taking a reference probability $P_{0}$ such that $P_{0}^{\mathcal{X}, \mathcal{Z}}=P_{*}^{\mathcal{X}, \mathcal{Z}}$ and $P_{0 \mathcal{Z}}=P_{* \mathcal{Z}}$ we have that $\mathcal{L}_{\mathcal{R} \mid \mathcal{X}, \mathcal{Z}}^{P_{\theta} / P_{0}}=1$ a.s. Thus we have, without additional assumption, $\mathcal{L}_{\mathcal{O}}^{P_{\theta} / P_{0}}=\mathrm{E}_{P_{0}}\left[\mathcal{L}_{\mathcal{X} \mid \mathcal{Z}}^{P_{\mathcal{Z}} / P_{0} \mathcal{W}} \mid \mathcal{O}\right]$. If CAR(TCMP) holds $\mathrm{E}_{P_{0}}\left[\mathcal{L}_{\mathcal{X} \mid \mathcal{Z}}^{P_{\theta} \mathcal{Z} / P_{0 \mathcal{W}}} \mid \mathcal{O}\right]$ does not depend on $P_{0}^{\mathcal{W}}=P_{*}^{\mathcal{W}}$ nor on $P_{0 \mathcal{Z}}=P_{* \mathcal{Z}}$. That is, we can compute the exact likelihood that we would like to compute if we knew $P_{*}^{\mathcal{X}, \mathcal{Z}}$ and $P_{* \mathcal{Z}}$, without in fact knowing them.

We can adapt now the same reasoning as in the previous section for defining the risk function for choosing estimators in the case where there are explanatory variables. We assume that we have $n$ i.i.d. triples $\left(X_{i}, Z_{i}, R_{i}\right)$, where the stochastic processes $Z_{i}$ represent time-dependent explanatory variables. Assuming that CAR(TCMP) holds for $\left(W_{i}, R_{i}\right), i=1, \ldots, n$, with $W_{i}=\left(X_{i}, Z_{i}\right)$ and using the reduced model we have that $\mathcal{L}_{\mathcal{O}_{n+1}}^{P_{\theta} / P_{*}}=\mathrm{E}_{P_{*}}\left[\mathcal{L}_{\mathcal{X}_{n+1} \mid P_{n+1} / P_{*} \mathcal{W}} \mid \mathcal{O}_{n+1}\right]$ depends neither on $P_{*}^{\mathcal{X}, \mathcal{Z}}$ nor on $P_{* \mathcal{Z}}$. We define the loss function as before as $-\log \mathcal{L}_{\mathcal{O}_{n+1}}^{P_{\hat{\theta}} / P_{*}}=-\log \mathrm{E}_{P_{*}}\left[\mathcal{L}_{\mathcal{X}_{n+1} \mid P_{n+1} / P_{*} / \mathcal{W}}^{P_{n+1}}, \mathcal{O}_{n}\right]$ and the risk function as $E K L_{n}=\mathrm{E}_{P_{*}}\left[-\log \mathcal{L}_{\mathcal{O}_{n+1}}^{P_{\hat{\theta}} / P_{*}}\right]$.

\section{Choice between semi-parametric models: like- lihood cross-validation}

\subsection{Estimating $E K L_{n}$ by likelihood cross-validation}

Making the assumption that CAR(TCMP) holds for $\left(X_{i}, R_{i}\right), i=1, \ldots, n$ we use a reduced model based on a reference probability $P_{0}$; then the observed likelihood ratio is $\mathcal{L}_{\overline{\mathcal{O}}_{n} / P_{0}}^{P_{\theta}}$. If there are explanatory variables, we assume that CAR(TCMP) holds for $\left(W_{i}, R_{i}\right), i=1, \ldots, n$, we use the reduced model and we still denote the observed likelihood $\mathcal{L}_{\overline{\mathcal{O}}_{n}}^{P_{\theta} / P_{0}}$.

Now we are seeking an "estimator" for our criterion $E K L_{n}$. We consider the leave-one-out likelihood cross-validation criterion as a possible "estimator". It is defined as:

$$
L C V_{n}\left(\hat{\theta}(. ; .), \kappa, \overline{\mathcal{O}}_{n}\right)=-\frac{1}{n} \sum_{i=1}^{n} \log \mathcal{L}_{\mathcal{O}_{i}\left(\kappa, \overline{\mathcal{O}}_{n \mid i}\right)}^{P / P_{0}},
$$

where $\overline{\mathcal{O}}_{n \mid i}=\vee_{j \neq i} \mathcal{O}_{j}$.

A first property, bearing on expectation of $L C V$ is: 
Lemma $1 \mathrm{E}_{P_{*}}\left[L C V_{n}\left(\hat{\theta}(. ;),. \kappa, \overline{\mathcal{O}}_{n}\right)\right]=E K L_{n-1}(\hat{\theta}(. ;),. \kappa)-K L\left(P_{0}, P_{*}\right)$.

Proof. We have

$$
\begin{aligned}
\mathrm{E}_{P_{*}}\left[L C V_{n}\left(\hat{\theta}(. ; .), \kappa, \overline{\mathcal{O}}_{n}\right)\right] & =-\mathrm{E}_{P_{*}}\left[\log \mathcal{L}_{\mathcal{O}_{i}\left(\kappa, \overline{\mathcal{O}}_{n \mid i}\right)}^{P_{\hat{O}} / P_{0}}\right] \\
& =-\mathrm{E}_{P_{*}}\left[\log \mathcal{L}_{\mathcal{O}_{i}\left(\kappa, \overline{\mathcal{O}}_{n \mid i}\right)}^{P_{*}}-\log \mathcal{L}_{\mathcal{O}_{i}}^{P_{*} / P_{0}}\right] \\
& =E K L_{n-1}(\hat{\theta}(. ; .), \kappa)-K L\left(P_{0}, P_{*}\right) .
\end{aligned}
$$

So, using $L C V_{n}$ for the choice of $\kappa$ we are using an unbiased estimator of $E K L_{n-1}$ : indeed, since $K L\left(P_{0}, P_{*}\right)$ does not depend on $\kappa$, we have $\mathrm{E}_{P_{*}}\left[L C V_{n}\left(\hat{\theta}(. ;),. \kappa_{2}, \overline{\mathcal{O}}_{n}\right)\right]-\mathrm{E}_{P_{*}}\left[L C V_{n}\left(\hat{\theta}(. ;),. \kappa_{1}, \overline{\mathcal{O}}_{n}\right)\right]=E K L_{n-1}\left(\hat{\theta}(. ;),. \kappa_{2}\right)-$ $E K L_{n-1}\left(\hat{\theta}(. ;),. \kappa_{1}\right)$. Thus $L C V$ estimates a difference in $E K L$ without the assumption that the true probability belongs to the model. Moreover we conjecture that the optimal properties obtained by Hall (1987) and van der Laan, Dudoit \& Keles (2004) extend under certain assumptions to the general context considered here and that cross-validation will effectively be able to choose between semi-parametric multi-state models.

\subsection{Computational algorithm}

\subsubsection{Approximation of the solution of the penalized likelihood}

The penalized likelihood estimator $\hat{\theta}_{\kappa}$ is the set of functions and parameters which maximize $p l_{\kappa}(\theta)$. In general it is not possible to compute $\hat{\theta}_{\kappa}$ analytically so the $\hat{g}_{k}^{\kappa}$ are approximated, for instance by splines. With this approximation the optimisation problem becomes a standard maximization on a finite number of parameters. (Note that the number of knots in the spline representation is limited only by computational issues: the smoothness of the final estimator for the $g_{k}($.$) is controlled by \kappa$ in the penalized likelihood, not by the number of knots). Calling $\gamma$ the set of new parameters (including $\beta$ and the set of spline parameters) we are led to maximizing:

$$
p l_{\overline{\mathcal{O}}_{n}}=p l_{\overline{\mathcal{O}}_{n}}^{\kappa}(\gamma)=L_{\overline{\mathcal{O}}_{n}}^{\gamma}-J(\gamma, \kappa),
$$

where $L_{\overline{\mathcal{O}}_{n}}^{\gamma}=\log \mathcal{L}_{\overline{\mathcal{O}}_{n}}^{\gamma / P_{0}}$. We note $\hat{\gamma}=\hat{\gamma}\left(\overline{\mathcal{O}}_{n}, \kappa\right)=\operatorname{argmax}_{\gamma}\left(p l_{\overline{\mathcal{O}}_{n}}^{\kappa}(\gamma)\right)$.

\subsubsection{Approximation of $L C V$}

Since $L C V_{n}$ (that we will note simply $L C V$ from now on) is particularly computationally demanding when $n$ is large an approximate version $L C V_{a}$ 
has been proposed by O'Sullivan (1988) for estimation of the hazard function in a survival case and adapted by Joly et al. (2002) to the case of intervalcensored data in an illness-death model. We may still extend it to a general framework valid for any penalized likelihood depending on a vector of real parameters $\gamma$. We note $L C V=-n^{-1} \sum_{i=1}^{n} L_{\mathcal{O}_{i}}^{\hat{\gamma}_{i}}$, where $\hat{\gamma}_{-i}=\hat{\gamma}\left(\overline{\mathcal{O}}_{n \mid i}, \kappa\right)$. The first order development of $L_{\mathcal{O}_{i}}^{\hat{\gamma}}$ around $\hat{\gamma}$ yields:

$$
L_{\mathcal{O}_{i}}^{\hat{\gamma}_{-i}} \approx L_{\mathcal{O}_{i}}^{\hat{\gamma}_{i}}+\left(\hat{\gamma}_{-i}-\hat{\gamma}\right)^{T} \hat{d}_{i}
$$

where $\hat{d}_{i}=\frac{\partial L_{\mathcal{O}_{i}}^{\gamma}}{\partial \gamma} \mid \hat{\gamma}$. The first order development of $\frac{\partial p l_{\overline{\mathcal{O}}_{n \mid i}}^{\gamma}}{\partial \gamma} \mid \hat{\gamma}_{-i}$ gives:

$$
\hat{\gamma}_{-i}-\hat{\gamma} \approx-H_{p l_{\overline{\mathcal{O}}_{n \mid i}}^{-1}} \frac{\partial p l_{\overline{\mathcal{O}}_{n \mid i}}^{\gamma}}{\partial \gamma} \mid \hat{\gamma}
$$

where $H_{p l_{\overline{\mathcal{O}}_{n \mid i}}}=\frac{\partial^{2} p l_{\overline{\mathcal{O}}_{n \mid i}}}{\partial \gamma^{2}} \mid \hat{\gamma}$, and more generally $H_{g}=\frac{\partial^{2} g}{\partial \gamma^{2}} \mid \hat{\gamma}$. At first order $H_{p l_{\overline{\mathcal{O}}_{n \mid i}}} \approx H_{p l_{\overline{\mathcal{O}}_{n}}}$. From the equality $p l_{\overline{\mathcal{O}}_{n}}(\gamma)=p l_{\overline{\mathcal{O}}_{n \mid i}}(\gamma)+L_{\mathcal{O}_{i}}^{\gamma}$ we deduce by taking derivatives:

$$
0=\frac{\partial p l_{\overline{\mathcal{O}}_{n \mid i}}^{\gamma}}{\partial \gamma} \mid \hat{\gamma}+\hat{d}_{i}
$$

which finally yields:

$$
\hat{\gamma}_{-i}-\hat{\gamma} \approx H_{p l_{\overline{\mathcal{O}}_{n}}}^{-1} \hat{d}_{i}
$$

which inserted in (6) gives:

$$
L_{\overline{\mathcal{O}}_{i}}^{\hat{\gamma}_{-i}} \approx L_{\overline{\mathcal{O}}_{i}}^{\hat{\gamma}}+\hat{d}_{i}^{T} H_{p l_{\overline{\mathcal{O}}_{n}}}^{-1} \hat{d}_{i}
$$

Substituting this expression in the expression of $L C V$ we obtain:

$$
L C V \approx L C V_{a_{1}}=-n^{-1}\left[L_{\overline{\mathcal{O}}_{n}}^{\hat{\gamma}}+\sum_{i=1}^{n} \hat{d}_{i}^{T} H_{p l_{\overline{\mathcal{O}}_{n}}}^{-1} \hat{d}_{i}\right]
$$

Using the fact that both $n^{-1} \sum_{i=1}^{n} \hat{d}_{i} \hat{d}_{i}^{T}$ and $-n^{-1} H_{L_{\overline{\mathcal{O}}_{n}}}$ tend towards the individual information matrix $I=-\mathrm{E}_{P_{*}}\left(H_{L_{\mathcal{O}_{i}}}\right)$ we get another approximation:

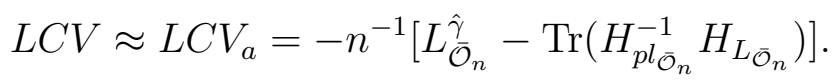

This expression looks like an AIC criterion and there are arguments to interpret $\operatorname{Tr}\left[H_{p l_{\overline{\mathcal{O}}_{n}}}^{-1} H_{L_{\overline{\mathcal{O}}_{n}}}\right]$ as the model degree of freedom. For instance, if there 
is no penalty $(J=0), H_{p l_{\overline{\mathcal{O}}_{n}}}=H_{L_{\overline{\mathcal{O}}_{n}}}$ so that the correction term in $L C V_{a}$ reduces to $\operatorname{dim}(\gamma)$, that is $L C V_{a}$ reduces to AIC.

If $\kappa$ is a scalar minimization of $L C V_{a}$ can be done by standard line-search algorithms; if it is a vector, a grid algorithm can be used (Joly et al., 2002).

\section{Simulation study}

\subsection{Description and main result}

We did a simulation study to illustrate the ability of $L C V$ to choose the right model structure; the possibilities were the Markov structure and the semiMarkov current-state structure, respectively models $\mathcal{M}_{1}$ and $\mathcal{M}_{2}$ in section 2.2. The best model is not always the right model, especially in the case where the right model is larger than alternative models. Here, the model 1 and model 2 structures are of similar complexities, so we think that the right model should be the best model.

We considered two particular models (or probability measures, $M_{1} \in \mathcal{M}_{1}$ and $M_{2} \in \mathcal{M}_{2}$ ). For both $M_{1}$ and $M_{2}$ the transition intensities toward illness, $\alpha_{01}(t)$, and death, $\alpha_{02}(t)$, were taken equal to the hazard function of Weibull distributions, namely: $p \gamma^{p} t^{p-1}$ with parameters $(p=2.4 ; \gamma=0.05)$ and $(p=2.5 ; \gamma=0.06)$ respectively. Models $M_{1}$ and $M_{2}$ differed by the intensity of $N_{2}$ for $t>T_{1}$, that is the mortality rate of diseased. For $M_{1}$ the mortality rate was defined by $h(t)$ and was a Weibull hazard function with parameters $(p=2.6 ; \gamma=0.08)$; for $M_{2}$ it was defined by $g\left(t-T_{1}\right)$ which was equal to a Weibull hazard function with parameters $(p=1.5 ; \gamma=0.2)$. For each subject we generated an ignorable TCMP observation scheme by generating $R_{1}$ and $R_{2}$ independently from $N_{1}$ and $N_{2}$. In intuitive terms $R_{1}$ and $R_{2}$ were constructed to represent a situation where $N_{1}$ was observed at discrete times and $N_{2}$ was observed in continuous time and possibly rightcensored (the same as in the application). For each subject we generated visit times $V_{j}$ at which $N_{1}$ was observed as $V_{j}=V_{j-1}+2+3 U_{j}$, where the $U_{j}$ 's were independent uniform $[0,1]$ random variables and observation of both $N_{1}$ and $N_{2}$ was right-censored by a variable $C$ which had a uniform distribution on $[2,52]$. We had $R_{1}(t)=1_{\{t<C\}} \sum_{j=1} 1_{\left\{t=V_{j}\right\}}$ and $R_{2}(t)=1_{\{t<C\}}$. To take into account the discrete-time observation scheme on one component we used formula (13) of Commenges \& Gégout-Petit (2006).

We generated 100 replicas of samples $N_{1}$ and $N_{2}$ from $M_{1}$ and $M_{2}$; each 
sample had $n=1000$ subjects. For each replica, the three functions determining the model $\left(\alpha_{01}(),. \alpha_{02}(\right.$.$) for both models and h($.$) for M_{1}$ and $g()$. for $M_{2}$ ) were estimated by penalized likelihood while the parameter $\hat{\eta}$ (determining model structure) and the smoothing coefficients $\hat{\kappa}=\left(\hat{\kappa}_{1}, \hat{\kappa}_{2}, \hat{\kappa}_{3}\right)$ which minimized $L C V$ were determined.

The first result is that when the Markov model was generated it was chosen in 99 cases out of 100; when the semi-Markov model was generated it was chosen in 93 cases out of 100 . This shows that $L C V$ does a good job in picking the right model structure. Table 1 shows the distance in term of the risk $E K L$ (the avrage Kullback-Leibler loss is an estimate of $E K L$ ) between the estimated models and the true model: choosing the model structure by $L C V$ incurs a very slight additional risk (of order $10^{-4}$ ) as compared to knowing the true model but a lower risk as compared to choosing the wrong model; in the latter case the additional risk is of order $10^{-2}$.

This result must not be falsely interpreted. First the discrimination properties of $L C V$ depends on many parameters and particularly on the quantity of information available in the samples. Second and even more important the aim of estimator choice is not to choose the right model but to choose the best estimator. The choice between the two structures depends on how "far" the two models are. If the models are "close" it is of course more difficult to discriminate between them, but at the same time it becomes less important to choose the right one. For instance the homogeneous Markov model belongs to both structures so it is possible by small perturbations of this model to construct two models, one Markov and one semi-Markov, which are very near in term for instance of Kullback-Leibler divergence.

\subsection{Study of the variability of $L C V$}

In this section we exploit the above simulation study to explore the variability of $L C V$. We have estimated from the 100 replicas from $M_{1}$ the density of $L C V(\hat{\kappa})$ assuming $\mathcal{M}_{1}$ and assuming $\mathcal{M}_{2}$. The upper-left panel of Figure 2 displays these estimated densities: there seems to be little difference between the two, so one may wonder whether $L C V$ can be of any use for choosing between the two model structures. However when we look at the density of the difference between $L C V(\hat{\kappa})$ for $\mathcal{M}_{1}$ and $\mathcal{M}_{2}$ we see that most of the mass is in the negative values, so that most of the time the true model $\mathcal{M}_{1}$ will be chosen. Similarly the lower panels show the estimated densities of $L C V$, assuming $\mathcal{M}_{1}$, for two different values $\kappa_{1}$ and $\kappa_{2}$ of the smoothing 
coefficient. Here the two densities are nearly undistinguishable while the density of the difference is clearly shifted toward positive values. Another way to examine this issue is to look at the standard deviations of $\operatorname{LCV}\left(\kappa_{1}\right)$, $L C V\left(\kappa_{2}\right)$ and $L C V\left(\kappa_{2}\right)-L C V\left(\kappa_{1}\right)$ : we estimated these values (under $\mathcal{M}_{1}$ ) to be $0.048,0.048$ and 0.0024 respectively. Thus the standard deviation of the difference is about twenty times less than that of $L C V$ for $\kappa_{1}$ or $\kappa_{2}$. This explains why $L C V$ does a good job in model choice in spite of its large variability.

\subsection{Quantitative interpretation of Kullback-Leibler di- vergences}

For practical use of the method proposed in this paper it is important to have an idea of whether a particular $E K L$ value, or a difference of $E K L$ values or their $L C V$ estimators are large or not. As in more conventional situations we must distinguish the interpretational issue from statistical issues. For instance in the conventional situation of a regression parameter the statistical issues beyond the point estimation of the parameter are to test the hypothesis of a null value of the parameter and to give a confidence interval for this parameter; the interpretational issue is to be able to assess the importance of the effect on the variable of interest. For instance in an epidemiological application using a Cox model we would consider the exponential of the parameter and interpret it as a relative risk, considering that a value of 1.1, 2, 5 would correspond to a small, moderate, large increase of risk respectively. We would like have a guide toward such an interpretation when manipulating $E K L$ values.

Since $E K L$ is an expected Kullback-Leibler divergence it can be interpreted as a Kullback-Leibler divergence. So let us try to interpret $K L\left(\tilde{P}, P_{*}\right)$. If we consider that $P_{*}$ is the true probability this means that we will make errors by evaluating the probability of an event $A$ by $\tilde{P}(A)$ rather than by $P_{*}(A)$. For instance we may evaluate the relative error $r_{e}\left(\tilde{P}(A), P_{*}(A)\right)=$ $\frac{P_{*}(A)-\tilde{P}(A)}{P_{*}(A)}$. Consider the typical event on which $\tilde{P}(A)$ will be under-evaluated defined as: $A=\left\{\omega: \mathcal{L}^{\tilde{P} / P_{*}}<1\right\}$. In order to obtain a simple formula relating $K L\left(\tilde{P}, P_{*}\right)$ to the error on $P_{*}(A)$ we consider the particular case $P_{*}(A)=1 / 2$ and $\mathcal{L}^{\tilde{P} / P_{*}}$ constant on $A$ and $A^{C}$ (or equivalently we compute $K L$ for a likelihood defined on $\sigma(A))$. In that case we easily find: $r_{e}\left(\tilde{P}(A), P_{*}(A)\right)=\sqrt{1-e^{-2 K L\left(\tilde{P}, P_{*}\right)}} \approx \sqrt{2 K L\left(\tilde{P}, P_{*}\right)}$, the approximation be- 
ing valid for small $K L$ value. For $K L$ values of $10^{-1}, 10^{-2}, 10^{-3}, 10^{-4}$, we find that $r_{e}\left(\tilde{P}(A), P_{*}(A)\right)$ is equal to $0.44,0.14,0.045$ and 0.014 , errors that we may qualify as "large", "moderate", "small" and "negligible".

As an example the $K L$ divergence of a double exponential relative to a normal distribution with same mean and variance is of order $10^{-1}$ leading to a "large" $r_{e}\left(\tilde{P}(A), P_{*}(A)\right)$. In the previous simulation study we have found that choosing the wrong model leads to an increase of the risk of order $10^{-2}$ which is "moderate", while choosing the model by $L C V$ leads to and increase of order $10^{-4}$ which may be qualified as "negligible".

\section{Application on dementia}

We illustrate the use of this general approach using the data of the Paquid study (Letenneur et al., 1999), a prospective cohort study of mental and physical aging that evaluates social environment and health status. The target population consists of subjects aged 65 years and older living at home in southwestern France. The diagnosis of dementia was made according to a two-stage procedure: the psychologist who filled the questionnaire screened the subjects as possibly demented according to DSM-III-R or not; subjects classified as positive were later seen by a neurologist who confirmed (or not) the diagnosis of dementia and made a more specific diagnosis, assessing in particular the NINCDS-ADRDA criteria for Alzheimer's disease. Subjects were re-evaluated 1, 3, 5, 8, 10 and 13 years after the initial visit. Subjects already demented at the initial visit were removed from the sample, a selection condition which is easily taken into account by using a conditional likelihood as mentioned in Commenges \& Gégout-Petit (2006). The sample consisted of 3673 subjects, 1540 men and 2133 women. Previous work (Commenges et al., 2004) has shown that the effect of gender on the risk of dementia is neither multiplicative nor additive; in fact the dynamics of ageing is so different between men and women that it is safer to perform completely separate analyses. For the purpose of this illustration we analyzed only women. During the 13 years of follow-up 396 incident cases of dementia and 835 deaths were observed. We wish to jointly model dementia and death, an approach conventionally referred to as the illness-death model; the model can be graphically described as in figure 1 , where the mortality rate of demented is noted $\alpha_{12}\left(t, t-T_{1}\right)$ to emphasize the fact that it may depend on both age $t$ and time since onset of dementia $t-T_{1}$; we assume 
that the transition intensities do not depend in addition on universal (or calendar) time. Note that dementia is observed in discrete time while death is observed in continuous time. One effect of the observation scheme is that we miss a certain number of dementia cases: we do not observe a dementia case which has happened when the subject develops dementia and dies between two planned visits. This scheme of observation and the likelihood for it are explained heuristically in Commenges et al. (2004) and rigorously in Commenges \& Gégout-Petit (2006).

We tried the three model structures depicted in section 2.2. We took as reference probability the homogeneous Markov model fitted to the data. Thus $L C V$ estimated the change in EKL when going from the homogeneous Markov maximum likelihood estimator to another estimator. The values of the best $L C V$ criteria for the different model structures were: Nonhomogeneous Markov model $\left(\mathcal{M}_{1}\right)$ : -0.2182 ; Current state model $\left(\mathcal{M}_{2}\right)$ : 0.2100; Excess mortality model $\left(\mathcal{M}_{3}\right)$ : -0.2180 . This means for instance that the best penalized likelihood estimator in the non-homogeneous model has an estimated expected Kullback-Leibler divergence $(E K L)$ relative to the true model which is smaller by 0.2182 than the homogeneous Markov estimator. The best $L C V$ was found for the non-homogeneous Markov model. However the best "excess mortality" estimator is not far from the best nonhomogeneous estimator, while the current state estimator seems to be farther. In terms of the interpretation of section 7.3 the difference between $\mathcal{M}_{2}$ and $\mathcal{M}_{1}$ is moderate while that between $\mathcal{M}_{3}$ and $\mathcal{M}_{1}$ is negligible.

We compared graphically the best estimators found for the three model structures considered. Figure 3 shows the three estimators for the age-specific incidence of dementia $\left(\alpha_{01}\right)$ and the mortality rates of non-demented respectively: the three estimators are very close for incidence of dementia; there is a certain difference between the Markov model and the two semi-Markov models for mortality rates of non-demented above 90 . Figure 4 displays the three estimators of the age-specific mortality rates of demented for different ages at onset of dementia, respectively 70, 80 and 90 . Here the patterns are different although the magnitude of these estimators are similar. In particular the current-state estimator is the same for the three ages at onset (by assumption) while we see a marked increase of mortality for age at onset of 90 in the non-homogeneous Markov estimator. From a qualitative point of view we may say that the three estimators agree for ages at onset of 70 and 80: the mortality rate for demented women does not vary much either with time since onset of dementia or with age at onset, and is around 0.2. 


\section{Conclusion}

We have extended the expected Kullback-Leibler risk function $(E K L)$ for estimator choice from generally coarsened observations of a stochastic process, including in the case of explanatory variables. We have suggested that this could be used for choosing both smoothing coefficients and model structure; we have suggested that $E K L$ could be approached by $L C V$ and we have given a general approximation formula for the leave-one-out $L C V$. The simulation presented showed that the $L C V$ did a good job in discriminating between model structures. The approach was illustrated in the problem of choosing between different additive illness-death models. The approach is in fact quite general and could be applied for instance to the choice between additive and multiplicative models.

Other choices might have been done: other loss functions, families of estimators and ways of estimating the risk function might have been chosen. However the choices we have done for the different components of the approach are adapted to the problem and fit well together. For instance the CAR(TCMP) assumption allows us to eliminate the nuisance parameters from the chosen loss function; $L C V$ is a natural estimator of $E K L$; penalized likelihood yields a flexible family of smooth estimators for which an approximation of $L C V$ can easily be computed. The approach yields an operational tool for exploring complex event histories, for instance in the domain of ageing.

There are many open problems and useful developments would be: finding a better algorithm for minimizing $L C V$ over multiple smoothing parameters; studying the variance of $L C V$ (see Bengio \& Grandvalet, 2004); finding asymptotic properties of the estimators chosen by minimizing $L C V$.

\section{References}

Aalen, O. (1978). Nonparametric inference for a family of counting processes. Ann. Statist. 6, 701-726.

Aalen, O., Borgan, O. \& Fekjaer (2001). H. Covariate Adjustment of Event Histories Estimated from Markov Chains: The Additive Approach. Biometrics 57, 993-1001.

Aalen, O. \& Johansen S. (1978). An empirical transition matrix for non- 
homogenous Markov chains based on censored observations. Scand. J. Statist. 5, 141-150.

Andersen, P.K, Borgan Ø, Gill RD \& Keiding N. (1993). Statistical Models Based on Counting Processes. New-York: Springer-Verlag.

Bengio, Y. \& Grandvalet, Y. (2004). No unbiased estimator of the variance of the K-fold cross-validation. Journal of Machine Learning Research 5, 1089-1105.

Commenges, D. \& Gégout-Petit, A., (2005). Likelihood inference for incompletely observed stochastic processes: ignorability conditions. arXiv:math.ST/0507151.

Commenges, D. \& Gégout-Petit, A. (2006). Likelihood for generally coarsened observations from multi-state or counting process models. Scand. J. Statist., in press.

Commenges, D. \& Joly, P. (2004). Multi-state model for dementia, institutionalization and death. Commun. Statist. A 33, 1315-1326.

Commenges, D., Joly, P., Letenneur, L. \& Dartigues, JF. (2004). Incidence and prevalence of Alzheimer's disease or dementia using an Illness-death model. Statist. Med. 23, 199-210.

Cox, D. \& O'Sullivan, F. (1990). Asymptotic analysis of penalized likelihood and related estimators. Ann. Statist. 18, 1676-1695.

Eggermont, P. \& LaRiccia, V. (1999). Optimal convergence rates for Good's nonparametric likelihood density estimator. Ann. Statist. 27, 1600-1615.

Eggermont, P. \& LaRiccia, V. (2001). Maximum penalized likelihood estimation. New-York: Springer-Verlag.

Gill, R. D., van der Laan, M. J. \& Robins, J.M. (1997). Coarsening at random: characterizations, conjectures and counter-examples, in: State of the Art in Survival Analysis, D.-Y. Lin \& T.R. Fleming (eds), Springer Lecture Notes in Statistics 123, 255-294.

Good, I.J. \& Gaskins, R.A. (1971). Nonparametric roughness penalty for probability densities. Biometrika 58, 255-277.

Gu, C. (1996). Penalized likelihood hazard estimation: a general procedure. 
Statistica Sinica 6; 861-876.

Hall, P. (1987). On Kullback-Leibler loss and density estimation. Ann. Statist. 15, 1491-1519.

Hougaard, P. (2000). Analysis of multivariate survival data. New York: Springer.

Jacod, J. (1975). Multivariate point processes: predictable projection; RadonNikodym derivative, representation of martingales. Z. Wahrsch. verw. Geb. 31, 235-253.

Joly, P. \& Commenges, D. (1999). A penalized likelihood approach for a progressive three-state model with censored and truncated data: Application to AIDS. Biometrics 55, 887-890.

Joly, P., Commenges, D., Helmer, C. \& Letenneur, L. (2002). A penalized likelihood approach for an illness-death model with interval-censored data: application to age-specific incidence of dementia. Biostatistics 3, 433- 443.

Kallenberg, O. (2001). Foundations of modern probabilities. Springer Verlag, New-York.

Kooperberg C. \& Clarkson DB. (1997). Hazard regression with intervalcensored data. Biometrics 53, 1485-1494.

Lagakos, S.W., Sommer, C.J. \& Zelen, M. (1978). Semi-Markov models for partially censored data. Biometrika $\mathbf{6 5}, 311-317$.

Le Cam, L. \& Yang, G. (2000). Asymptotics in Statistics. New-York: Springer-Verlag.

Letenneur, L., Gilleron, V., Commenges, D., Helmer, C., Orgogozo, JM. \& Dartigues JF. (1999). Are sex and educational level independent predictors of dementia and Alzheimer's disease ? Incidence data from the PAQUID project. J. Neurol. Neurosurg. Psychiatr. 66, 177-183.

Liquet, B. \& Commenges, D. (2004). Estimating the expectation of the loglikelihood with censored data for estimator selection. Lifetime Data Analysis 10, 351-367.

Liquet, B., Saracco, J. \& Commenges, D. (2006). Selection between pro- 
portional and stratified hazards models based on expected log-likelihood. Computational Statistics, in press.

Liquet, B., Sakarovitch, C \& Commenges, D.(2003). Bootstrap choice of estimators in parametric and semi-parametric families: an extension of EIC. Biometrics 59, 172-178.

Müller, HG. \& Stadtmüller, U. (2005). Generalized functional linear models. Ann. Statist. 33, 774-805.

O'Sullivan, F. (1988). Fast computation of fully automated log-density and log-hazard estimators. SIAM Journal on Scientific and Statistical Computing 9, 363-379.

Ramlau-Hansen, H. (1983). Smoothing counting process intensities by means of kernel functions. Ann. Statist. 11, 453,466.

Scheike, T. (2001). A generalized additive regression model for survival analysis. Ann. Statist. 29, 1344-1380.

van der Laan, M. \& Dudoit, S. (2003). Unified cross-validation methodology for selection among estimators and a general cross-validated adaptive epsilon-net estimator: finite sample oracle inequalities and examples. Berkeley Division of Biostatistics working paper series, paper 130.

van der Laan, M., Dudoit, S. \& Keles, S. (2004). Asymptotic optimality of likelihood-based cross-validation. Statistical Applications in Genetics and Molecular Biology 3, Issue 1, article 4.

Daniel Commenges, INSERM U 875; Université Victor Segalen Bordeaux 2, 146 rue Léo Saignat, Bordeaux, 33076, France

E-mail: daniel.commenges@isped.u-bordeaux2.fr 


\begin{tabular}{l|ccc}
\hline replications $=100$ & & $\overline{K L}$ & \\
sample size $=1000$ & $\mathcal{M}_{1}$ & $\mathcal{M}_{2}$ & $\mathcal{M}_{1}$ or $\mathcal{M}_{2}$ \\
\hline true model $\mathcal{M}_{1}$ & $0.00489(0.00024)$ & $0.02918(0.00024)$ & $0.00515(0,00035)$ \\
\hline true model $\mathcal{M}_{2}$ & $0.10335(0.00018)$ & $0.09675(0.00019)$ & $0.09719(0,00025)$ \\
\hline \hline
\end{tabular}

Table 1: Average Kullback-Leibler loss $\overline{K L}$ and the corresponding standard errors (numbers in the parentheses) for estimators chosen by $L C V$ 


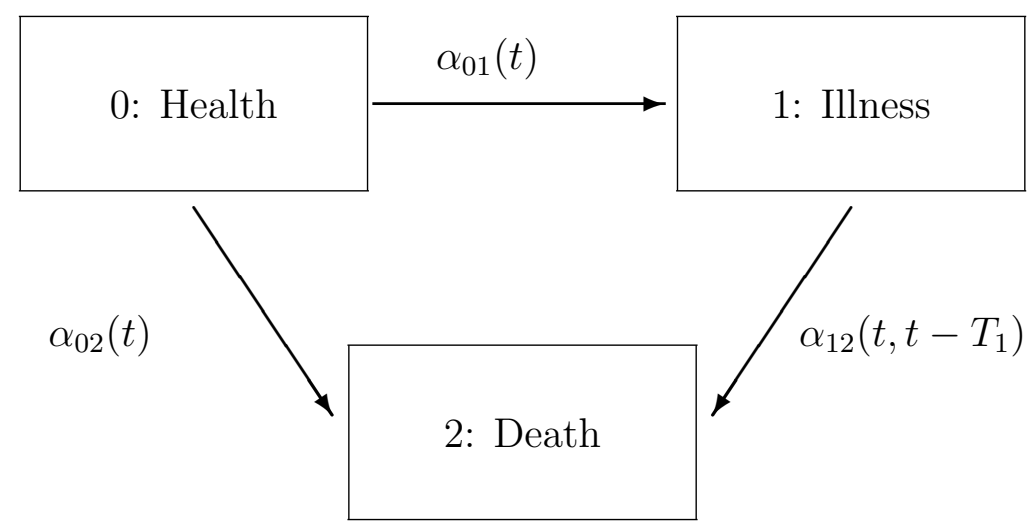

Figure 1: The illness-death model. $t$ : age; $T_{1}$ : age of onset of illness. 

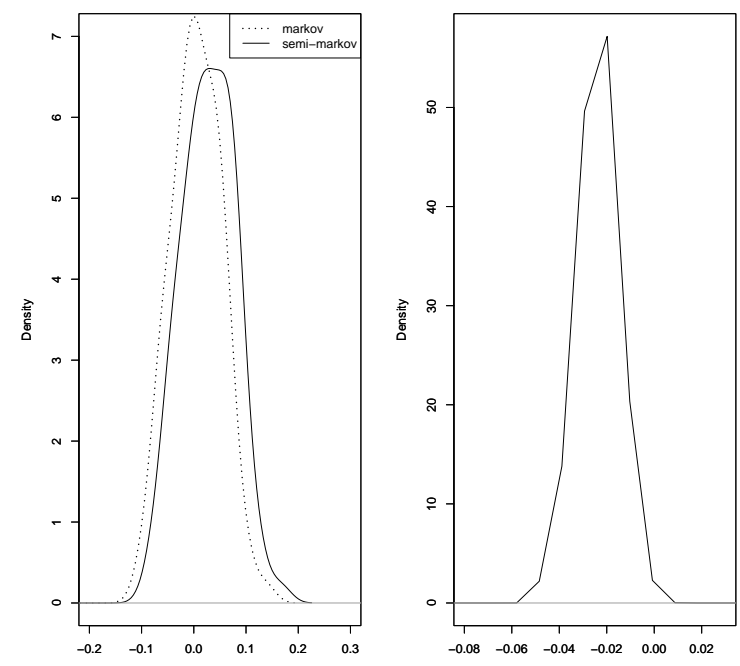

a
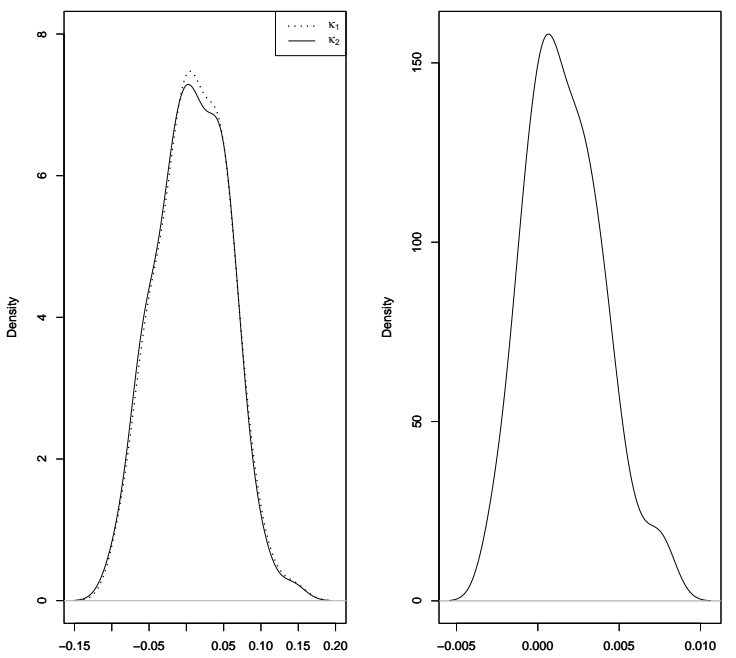

$\mathrm{b}$

Figure 2: Kernel density estimation of $L C V$ (left) and of differences of $L C V$ (right) for a) Markov and semi-Markov choices, b) for two different values of the smoothing parameter; in all cases the true model is Markov. 

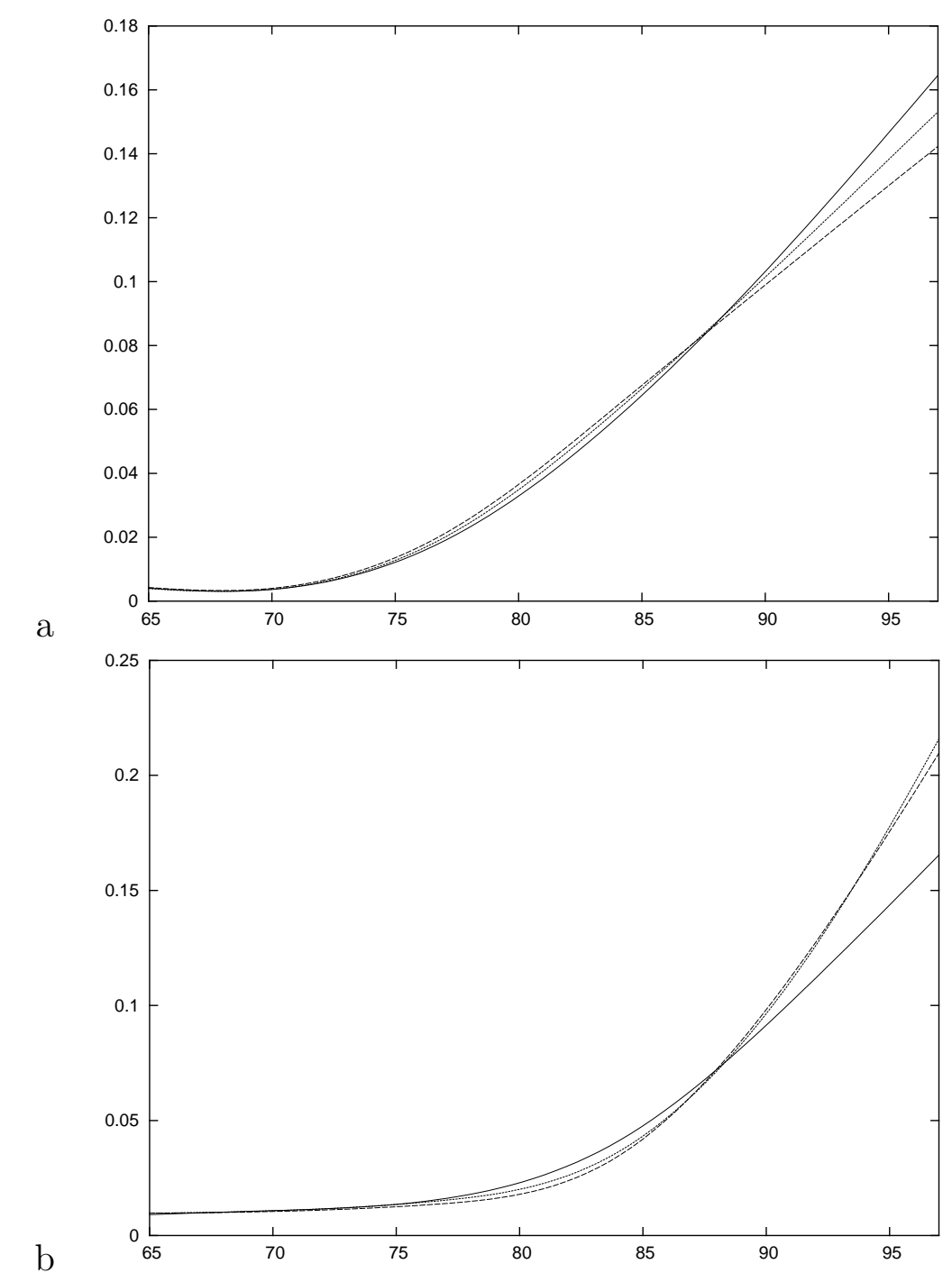

Figure 3: Incidence of dementia (a) and mortality (b) for non-demented women for the three models. Continuous line: non-homogeneous Markov model; dashed line: current state model; dotted line: excess mortality model 

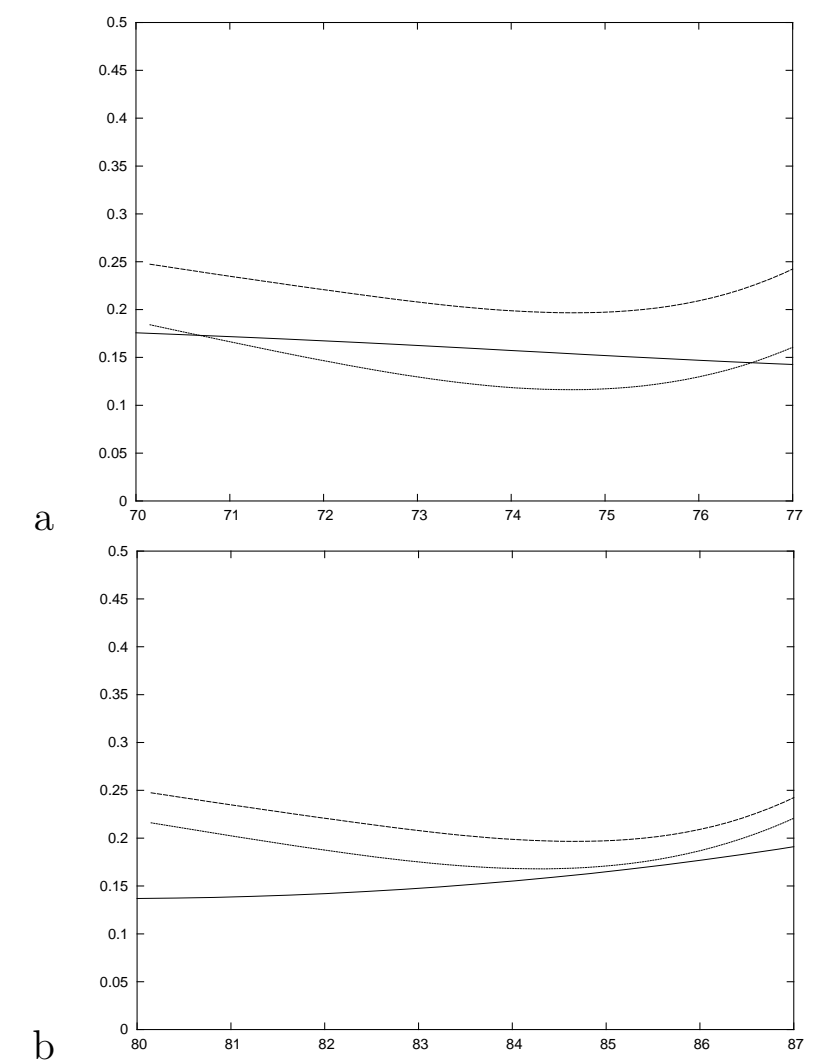

$\mathrm{b}$

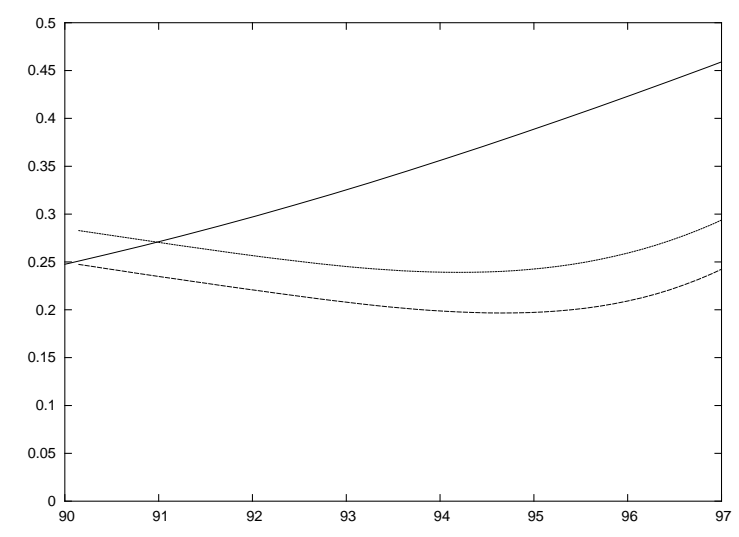

Figure 4: Mortality of demented women for the three models: Continuous line: non-homogeneous Markov model; dashed line: current state model; dotted line: excess mortality model. Age at onset of dementia: a): 70; b): 80; c): 90 . 